\title{
Especificaciones europeas para los estándares globales en educación médica
}

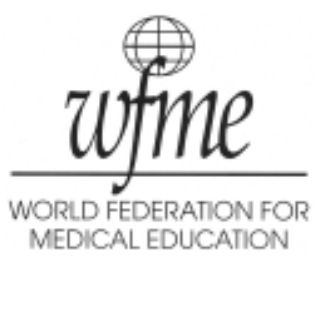

La expansión del proceso de globalización que se ha producido en el campo de la medicina, puesta de manifiesto por el incremento del intercambio de estudiantes de medicina, la emigración de médicos y la educación trans-fronteriza ha acentuado la necesidad de disponer de unos estándares en educación médica. Los estándares son también importantes de cara a afrontar los problemas y los retos nacionales. El impacto del programa de los Estándares Globales de la Federación Mundial para la Educación Médica (World Federation for Medical Education, WFME) ${ }^{1}$ concuerda con estas premisas.

Los Estándares Globales para la Educación Médica en sus tres fases ${ }^{2-4}$, publicados en 2003, fueron elaborados para que pudieran ser usados a nivel nacional, regional e institucional como un sistema de referencia en los procesos de reforma institucional y para el reconocimiento / acreditación de las instituciones educativas y sus programas.

Era lógico por ello que MEDINE, la red temática sobre Educación Médica en Europa ${ }^{5}$, subvencionada por la Comisión de la Unión Europea, decidiera incluir en sus objetivos, actividades dirigidas a asegurar la calidad y a establecer estándares para la educación médica en la Región Europea.

Los objetivos del grupo de trabajo de MEDINE sobre Estándares para asegurar la calidad, liderado conjuntamente por la WFME y la Asociación de Facultades de Medicina en Europa, (Association of Medical Schools in Europe, AMSE), fueron estimular la mejora de los estándares para la educación médica, analizar la validez de los estándares en el contexto europeo y elaborar un conjunto de estándares para asegurar la calidad de la educación médica en Europa, trabajando sobre y adaptando existentes, como los Estándares Globales de la WFME.

El grupo de trabajo decidió considerar Europa en un sentido amplio, y encontró que la región relevante para la educación médica podría coincidir con el área geográfica correspondiente al Consejo de Europa. Al mismo tiempo, el grupo de trabajo era consciente de la creciente relación entre la Región Europea, definida de acuerdo con este criterio y los países vecinos y que cabía esperar en el futuro una expansión de la Región Europea en términos de relevancia para la educación médica y la movilidad de los médicos. Además el grupo de trabajo enfatizó que el establecimiento de estándares para Europa no debía crear una situación de aislamiento respecto a otras partes del mundo, ya que los estándares en educación médica deberían ser usados como un instrumento para salvaguardar la calidad de la profesión médica, y no representar una barrera innecesaria que impidiese la movilidad de médicos entre Europa y otras partes del mundo.

El grupo de trabajo también consideró otros factores como la diversidad de la educación médica en la Región y la necesidad de establecer estándares, incluyendo las necesidades especificas europeas determinadas por ejemplo por la Directiva Europea sobre el reconocimiento de calificaciones profesionales $^{6}$ y las consecuencias del Espacio Europeo de Educación Superior tal como se expresan en la Declaración y en el Proceso de Bolonia?.

Teniendo en cuenta todos estos aspectos, el grupo de Trabajo llegó a la conclusión de que no había rezones para mantener un nivel intermedio entre los estándares globales y los nacionales en la Región Europea. En consecuencia, rechazó la formulación de estándares separados para Europa y llegó a la conclusión de que bastaba con establecer especificaciones Europeas para los estándares globales de la WFME.

$\mathrm{Al}$ formular estas especificaciones, el grupo de trabajo consideró razonable modificar las líneas de división existente entre el nivel de estándares básicos o requerimientos mínimos y el nivel de estándares para el desarrollo de la calidad, usados en los Estándares Globales de la WFME. Considerando algunos de los estándares para el desarrollo de la calidad como estándares mínimos, se tomaba en consideración las condiciones generales sociales y económicas de la Región, en comparación con otras partes del mundo y también las recientes mejoras y los esfuerzos llevados a cabo para asegurar la calidad y el desarrollo de la educación médica en Europa, lo cual permitía establecer estándares de mayor nivel. Además, se añadieron algunos nuevos -pocos sorprendentemente- o algunos suplementarios. Debe señalarse que esta revisión desde la óptica europea, realizada por el grupo de trabajo de los Estándares de la WFME, puso de manifiesto que dichos Estándares no requerían en estos momentos una mayor revisión.

Los resultados del grupo de trabajo de MEDINE se publicaron en mayo de $2007^{8}$.

Las recomendaciones propuestas referidas a la aplicación de los Estándares en educación médica podrán ser usadas por la Comisión Europea, autoridades educativas o sanitarias nacionales, instituciones y organizaciones con responsabilidades en educación médica en sus esfuerzos para lograr asegurar la calidad y para la mejora de la educación médica a lo largo de su continuum en la Región Europea.

\section{Bibliografía}

1. World Federation for Medical Education (WFME). Global Standards Programme, 2007, http://www.wfme.org, (accessed 10 May 2007).

2. World Federation for Medical Education (WFME). Basic Medical Education. WFME Global Standards for Quality Improvement. Copenhagen, 2003, http://www.wfme.org, (accessed 10 May 2007).

3. World Federation for Medical Education (WFME).

Postgraduate Medical Education. WFME Global Standards for Quality Improvement. Copenhagen, 2003, ttp://www.wfme. org, (accessed 10 May 2007).

4. World Federation for Medical Education (WFME). Continuing Professional Development (CPD) of Medical Doctors. WFME Global Standards for Quality Improvement. Copenhagen, 2003, http://www.wfme.org, (accessed 10 May 2007).

5. MEDINE. The Thematic Network on Medical Education in Europe, 2007, http://www.bris.ac.uk/medine, (accessed 10 May 2007).

6. EU Directive 2005/36/EC of 7 September 2005 on the recognition of professional qualifications, 2005,

http://europa.eu.int/comm/internal_market/qualifications/future en.htm, (accessed 10 May 2007).

7. The Framework of Qualifications for the European Higher Education Area, 2005 http://www.bologna-bergen, (accessed 10 May 2007).

8. MEDINE. The Thematic Network on Medical Education in Europe. WFME Global Standards for Quality Improvement in Medical Education. European Specifications. Copenhagen, 2007. 